\title{
Variation in cue duration reveals top-down modulation of involuntary orienting to uninformative symbolic cues
}

\author{
BRADLEY S. GIBSON and TED A. BRYANT \\ University of Notre Dame, Notre Dame, Indiana
}

\begin{abstract}
This article reports three experiments in which the effects of cue duration on involuntary orienting to uninformative symbolic cues (arrows presented at fixation) were investigated. Experiment 1 showed that symbolic cues had less effect on involuntary orienting when they were presented for only $25 \mathrm{msec}$ than when they were presented for $200 \mathrm{msec}$ across a range of stimulus onset asynchronies. Experiment 2 suggested that the effect of cue duration on involuntary orienting was due primarily to top-down strategic factors, rather than to bottom-up stimulus factors, and Experiment 3 suggested that these strategic factors may involve differences in how the cue is processed. Altogether, the present findings are important because they emphasize the distinction between cue processing and the putative involuntary orienting that results from such processing in the symbolic-cuing paradigm. In so doing, the present results help resolve discrepant findings that have been reported across previous studies.
\end{abstract}

Contemporary research suggests that attention is organized into a number of interrelated processing networks that are widely distributed throughout the brain (Posner \& Petersen, 1990). Because high-level visual processing is limited in capacity, one critical function of attention is to select a subset of available information in the visual field for further processing. Such selection is thought to be carried out by the spatial-orienting network, which directs focal attention to various spatial locations and/or objects present in the visual field. The orientation of attention has been extensively studied over the past 2 decades, using the spatial-cuing paradigm (Posner, 1980; Posner, Snyder, \& Davidson, 1980). In this paradigm, spatial cues are used to direct attention to potential target locations, and both response time (RT) and accuracy have been measured to assess processing differences associated with attended and unattended stimuli.

In the present article, we address a recent controversy concerning a particular class of spatial cues that have been referred to as symbolic cues. Symbolic cues are stimuli that typically appear at fixation and refer to potential target locations indirectly, as for instance, when an arrow points to one of several potential target positions located in the visual periphery. Because these cues have the potential to influence processing of an ensuing target by directing attention from a central location to another, more peripheral location according to the symbolic meaning

The authors thank Tom Carr, Bernhard Hommel, Steve Luck, and an anonymous reviewer for their helpful comments on an earlier version of this article. Correspondence concerning this article should be addressed to B. S. Gibson, 118 Haggar Hall, Department of Psychology, University of Notre Dame, Notre Dame, IN 46556 (e-mail: bgibson@nd.edu). of the cue, they have also been called central, indirect, push, and endogenous cues. Symbolic cues can be contrasted with a more direct form of spatial cuing in which the cues appear at, or in close proximity to, potential target locations that are located away from central fixation in the peripheral visual field. In contrast to symbolic cues, direct cues do not require interpretation; rather, they have the potential to influence processing of an ensuing target by attracting attention directly to the location of the cue. For this reason, these cues have also been called peripheral, direct, pull, and exogenous cues.

Of particular interest in the present article is the question of whether a particular kind of symbolic cue - an arrow presented at fixation - can elicit involuntary shifts of attention within the spatial-cuing paradigm. Attention is said to be controlled in an involuntary fashion when it is allocated independently of the current goals and intentions of an observer, which is typically achieved in the spatialcuing paradigm by presenting cues that predict the location of the target only by chance (for a more detailed discussion of this issue, see Bacon \& Egeth, 1994; Folk, Remington, \& Johnston, 1992; Gibson \& Kelsey, 1998; Theeuwes, 1994; Yantis, 1993). For the past 20 years, the established view has been that symbolic cues are not capable of eliciting involuntary shifts of attention. The empirical basis for this conclusion can be traced to the seminal research of Jonides (1981; see especially Experiment 2), which suggested that observers could intentionally suppress orienting in response to an uninformative arrow cue presented at fixation, but not in response to an uninformative arrow cue presented in the visual periphery.

However, although Jonides's (1981) conclusion that symbolic arrow cues cannot elicit involuntary shifts of attention has stood more or less unchallenged for the past 
2 decades, this view has recently begun to change. For instance, evidence that at least some kinds of symbolic cues might be capable of resisting suppression came initially from research showing that the gaze direction of a centrally fixated face produced attentional cuing effects even when the direction in which the eyes were gazing was unpredictive of the target's location, suggesting that these cues might elicit an involuntary form of attentional orienting (Friesen \& Kingstone, 1998; see also, Driver et al., 1999; Friesen \& Kingstone, 2003; Hietanen, 1999; Kingstone, Friesen, \& Gazzaniga, 2000). At first glance, the apparent discrepancy between recent findings obtained with eye gaze cues and those obtained by Jonides with arrow stimuli may be interpreted as supporting the ecological significance of faces; however, contrary to Jonides's original findings, subsequent evidence has begun to accumulate that suggests that a variety of over learned symbolic cues, as well as facial cues, appear capable of eliciting involuntary shifts of attention.

More specifically, within the past few years, a wealth of behavioral evidence has accumulated to suggest that uninformative symbolic cues (such as arrows and spatial terms) can resist suppression under a wide range of different stimulus and task conditions (Eimer, 1997; Hommel, Pratt, Colzato, \& Godijn, 2001; Pratt \& Hommel, 2003; Ristic, Friesen, \& Kingstone, 2002; Tipples, 2002). Contrary to Jonides's (1981) initial findings, these more recent findings appear to provide compelling evidence that uninformative symbolic cues can elicit involuntary shifts of visual attention. In the present article, we sought to extend this growing body of research by attempting to better understand the discrepancy between the positive findings reported in these recent studies and the null findings reported by Jonides. For this reason, we have focused the present investigation on symbolic arrow cues. Although more recent studies have obtained evidence that symbolic arrow cues can elicit involuntary attentional orienting under a wide range of stimulus and task conditions, there is at least one potentially important stimulus factor - cue duration - that has not yet been systematically investigated. In particular, symbolic arrow cues were presented for only $25 \mathrm{msec}$ in Jonides's study, whereas arrow cues have tended to be presented for much longer durations (ranging from $75 \mathrm{msec}$ to the length of a trial) in more recent studies (Eimer, 1997; Hommel et al., 2001; Pratt \& Hommel, 2003; Ristic et al., 2002; Tipples, 2002). Moreover, studies in which cue duration has been varied (Hommel et al., 2001; Tipples, 2002) have tended to do so across experiments in which the variation in cue duration has been confounded with other significant stimulus or task variation, making it difficult to isolate the true effect of this variable. In the present article, we report experiments that were explicitly designed to investigate whether the duration of a symbolic arrow cue can affect its ability to elicit involuntary orienting. In so doing, our experiments are attempts to provide greater understanding of the symbolic control of visual attention.

\section{EXPERIMENT 1}

In Experiment 1, the cue duration ( $25 \mathrm{msec}$ ) used by Jonides (1981) was compared with a longer duration cue $(200 \mathrm{msec})$ that fell within the range of values previously shown to be effective (Eimer, 1997; Hommel et al., 2001; Pratt \& Hommel, 2003; Ristic et al., 2002; Tipples, 2002). On the basis of these previous findings, we therefore expected that the short-duration cues used in the present experiment would elicit less involuntary orienting (as reflected by the magnitude of the cue validity effect) than the long-duration cues would. Note, however, that the 25-msec cues used by Jonides were also paired with a relatively short cue-target stimulus onset asynchrony (SOA; $75 \mathrm{msec}$ ) in his study. We therefore thought it prudent to determine whether the difference in cue validity that was expected to be observed between the 25 - and the 200-msec arrow cues in the present experiment would hold across a variety of cue-target SOAs. Accordingly, in addition to cue duration, Experiment 1 also varied the SOA between the cue and the target, including values of 50,300 , and $550 \mathrm{msec}$. In addition, it is worth mentioning that the task used in the present experiment was very similar to the task used in Jonides's experiment. In particular, observers were required to discriminate the identity of a target letter $(\mathrm{H}$ or $\mathrm{U})$ that appeared among seven similar distractor letters. This relatively demanding visual search task was chosen because it has the potential to produce larger attentional-cuing effects than less demanding tasks do (such as when only a single stimulus appears in the target display; see, e.g., Experiments 3 and 4 in Hommel et al., 2001; Ristic et al., 2002; Tipples, 2002), which should optimize our ability to observe a difference between the 25-and the 200-msec cues, if such a difference exists.

\section{Method}

Participants. Forty undergraduates from the University of Notre Dame participated in this experiment in partial fulfillment of a course requirement. All the participants in the study reported normal or corrected-to-normal vision. None was aware of the purpose of the study.

Stimuli and Apparatus. Three separate displays were shown in Experiment 1. The fixation display consisted of a centrally located white fixation dot that was surrounded by eight gray boxes. The diameter of the fixation dot subtended $0.38^{\circ}$ of visual angle; the boxes subtended $2.67^{\circ} \times 1.62^{\circ}$ of visual angle and were located $3.81^{\circ}$ from the dot. The boxes were equally spaced around the circumference of an imaginary circle. The cue display was identical to the fixation display, except that the fixation dot was replaced by a white arrow that pointed to one of the eight peripheral boxes. The arrow subtended $0.48^{\circ} \times 1.34^{\circ}$ of visual angle. In the target display, a red letter appeared centered in each of the eight boxes, one of which was always the target. The two target letters were $\mathrm{H}$ and $\mathrm{U}$, and the distractor letters were A, C, E, F, L, P, and S. To ensure that the letters were visually similar, they were created by erasing various line segments from a block figure eight. The letters subtended approximately $1.05^{\circ} \times 0.96^{\circ}$ of visual angle. All the stimuli appeared on the black background of a standard CRT monitor viewed by the observer from a distance of approximately $60 \mathrm{~cm}$. This distance was held constant by a chin-head rest. Responses were recorded on a 
custom-made button box (Lafayette Instruments) and were recorded to the nearest millisecond. RTs and errors were recorded on a Dell Optiplex computer.

Procedure. The sequence of displays that appeared on each trial is depicted in Figure 1. Each trial in Experiment 1 began with the fixation display for $500 \mathrm{msec}$, and the observers were instructed to keep their eyes focused on the dot throughout each trial (although fixation was not explicitly monitored in the present experiments). The cue display then appeared for either 25 or $200 \mathrm{msec}$. Cue duration remained consistent within separate blocks of trials. Within each of these blocks, the cue preceded the target display by 50,300 , or $550 \mathrm{msec}$. Note that the fixation display reappeared between the cue display and the target display on those trials in which the cue-target SOA was greater than the cue duration. The cue pointed at the eventual location of the target on only $12.5 \%$ of the trials in this experiment (the valid trials), and it pointed at the eventual location of one of the seven distractors on the remaining $87.5 \%$ of the trials (the invalid trials). Thus, the cue indicated the location of the target only by chance in this experiment; for this reason, the cue was considered to be irrelevant, and the observers were explicitly instructed to ignore it. The target display remained present until response or until 2,000 msec had elapsed. The observers were instructed to press the left key on the response box when the $\mathrm{H}$ appeared and to press the right key when the $U$ appeared. The observers were instructed to respond as quickly as possible while also keeping error rates to a minimum. Each of the two trial blocks included 192 experimental trials, and each was preceded by a representative set of 16 practice trials. The order of the two trial blocks was counterbalanced across observers; the order of trials within each block was randomly determined for each observer.

\section{Results and Discussion}

Mean correct RTs in each of the two cue duration conditions are shown in Figure 2 as a function of SOA and validity. Trials on which the observers pressed the wrong key were treated as errors, as were trials on which RT was less than $200 \mathrm{msec}$ or greater than $2,000 \mathrm{msec}$. These error rates are listed in Table 1 as a function of cue duration, SOA, and validity. Overall, the error rates were relatively low in this experiment $(3.87 \%)$ and did not differ significantly across conditions (all $p \mathrm{~s}>.10$ or more). Thus, the observers did not appear to be sacrificing accuracy for speed in this experiment.

Mean correct RT was analyzed using a three-way repeated measures analysis of variance (ANOVA), with cue duration ( 25 vs. $200 \mathrm{msec}$ ), SOA (50 vs. $300 \mathrm{msec}$ vs. $550 \mathrm{msec}$ ), and validity (valid vs. invalid) as the three within-subjects factors. There was a main effect of SOA $\left[F(2,78)=24.80, M S_{\mathrm{e}}=3,883.62, p<.0001\right]$, which indicated that RTs generally decreased as SOA increased. There was also a main effect of validity $[F(1,39)=29.70$, $\left.M S_{\mathrm{e}}=1,3121.32, p<.0001\right]$, which indicated that RTs were significantly shorter in the valid condition than in the invalid condition. This finding is consistent with those in other recent studies (Eimer, 1997; Hommel et al., 2001; Pratt \& Hommel, 2003; Ristic et al., 2002; Tipples, 2002 ) and suggests that the symbolic arrow cues used in the present study were capable of eliciting an involuntary form of orienting. More important, the cue validity effect also interacted separately with SOA $[F(2,78)=4.05$, $\left.M S_{\mathrm{e}}=3,733.51, p<.025\right]$ and cue duration $[F(1,39)=$ $\left.5.87, M S_{\mathrm{e}}=47,780.28, p<.025\right]$ in this experiment. No other main effects or interactions approached significance in this experiment (all $p \mathrm{~s}>.50$ or more).

As can be seen in Figure 2, the magnitude of the cue validity effect (invalid RT - valid RT) increased from 35 to $68 \mathrm{msec}$ as SOA increased from 50 to $300 \mathrm{msec}$; however, there was no change in the magnitude of this

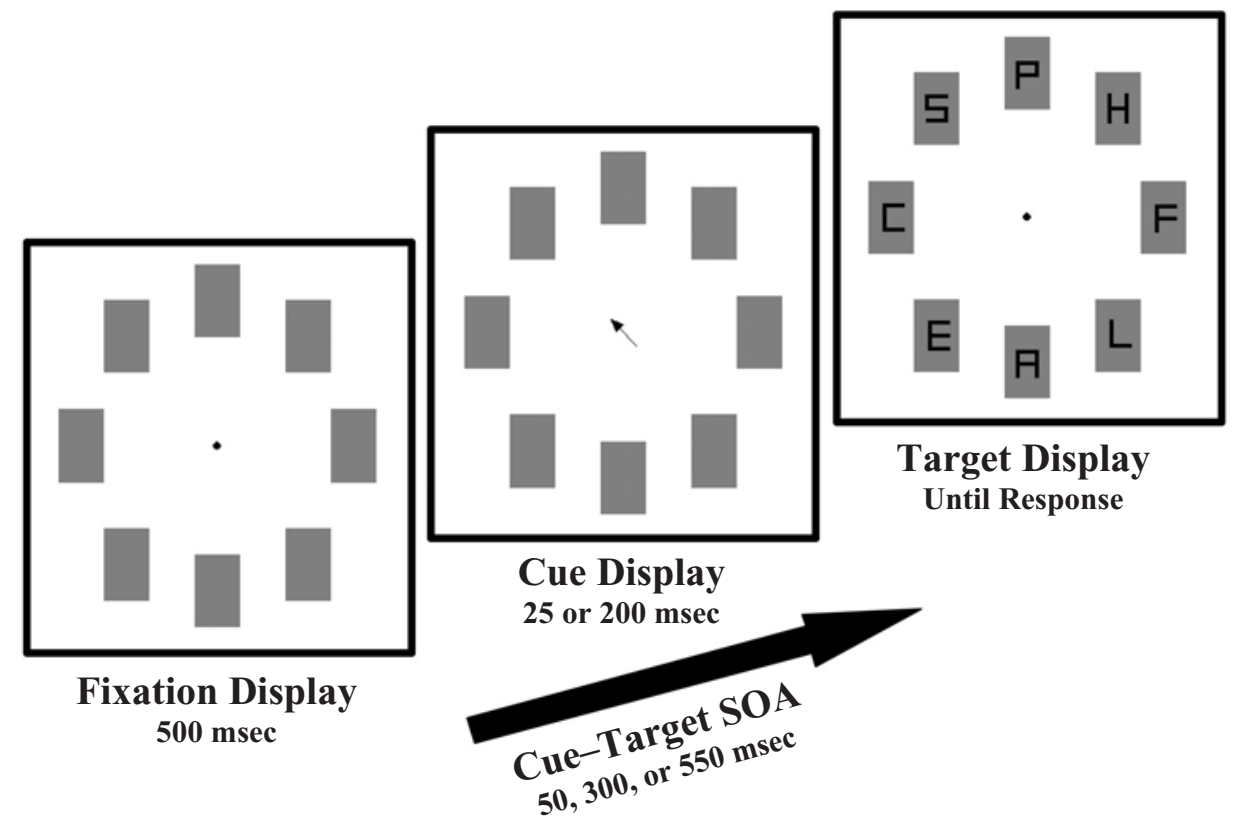

Figure 1. The display sequence that was shown on each trial in Experiment 1. Note that the letters appeared red in the actual displays. Note also that the fixation display reappeared between the cue display and the target display on those trials in which the cue-target stimulus onset asynchrony (SOA) was greater than the cue duration. 


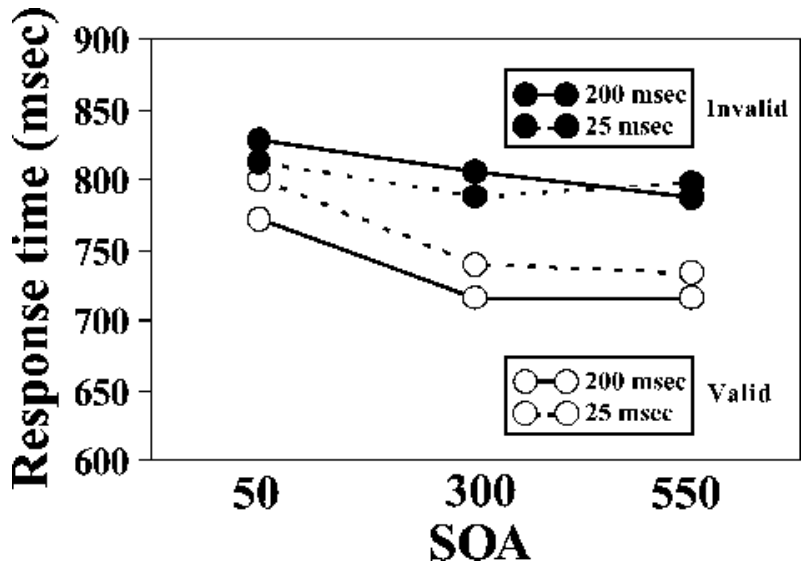

Figure 2. Mean correct response times shown as a function of cue duration, stimulus onset asynchrony (SOA, in milliseconds), and validity in Experiment 1.

effect as SOA increased further to $550 \mathrm{msec}$. This SOA $\times$ validity interaction suggests that shifting attention from the centrally located symbolic cue to the peripherally located target display is a time-consuming process and that approximately $300 \mathrm{msec}$ are required for attention to reach its maximal effect at the target location (Cheal \& Lyon, 1991; Müller \& Rabbitt, 1989; Pratt \& Hommel, 2003), although follow-up ANOVAs revealed that valid RTs were significantly shorter than invalid RTs at all three SOAs (all $p \mathrm{~s}<.005$ or less).

In addition, although the magnitude of the cue validity effect increased similarly across SOA in both the 25and the 200-msec cue duration conditions, as was expected, the magnitude of the cue validity effect was overall smaller (by an average of $30 \mathrm{msec}$ ) in the 25 -msec cue duration condition than in the 200-msec cue duration condition. This cue duration $\times$ validity interaction suggests that the 25-msec cues were consistently less effective at shifting attention than were the 200-msec cues, although it should once again be noted that the overall effect of cue validity was found to be significant in both the 25 -msec cue duration condition (overall cue validity effect $=42 \mathrm{msec}$ ) and the $200-\mathrm{msec}$ cue duration condition [overall cue validity effect $=72 \mathrm{msec} ; F(1,39)=$

Table 1

Error Rates (in Percentages) as a Function of Cue Duration, Stimulus Onset Asynchrony (SOA, in Milliseconds), and Validity in Experiment 1

\begin{tabular}{lccc}
\hline & \multicolumn{3}{c}{ SOA } \\
\cline { 2 - 4 } Validity & 50 & 300 & 550 \\
\hline \multirow{4}{*}{ Valid } & $25-\mathrm{msec}$ & Cue & Duration \\
Invalid & 5.18 & 1.88 & 3.75 \\
& 3.58 & 4.36 & 4.56 \\
Valid & 200 -msec & Cue Duration & \\
Invalid & 3.80 & 3.28 & 3.53 \\
\hline
\end{tabular}

$14.82, M S_{\mathrm{e}}=2,346.06, p<.0001$, and $F(1,39)=28.86$, $M S_{\mathrm{e}}=3,620.47, p<.0001$, respectively]. The significant effect of cue validity observed in the 25 -msec cue duration condition (averaged across the three SOA conditions) therefore appears to contradict the null effect of cue validity reported by Jonides (1981, Experiment 2). However, recall that the cue validity effects associated with Jonides's 25 -msec cues were confined to a single, relatively short (75-msec) cue-target SOA. Similarly, we also found a relatively small $(12-\mathrm{msec})$ and nonsignificant $[F(1,39)<1]$ effect of cue validity in the present experiment when the effects of our 25 -msec cues were analyzed exclusively at the shortest $(50-\mathrm{msec})$ SOA. In contrast, the cue validity effect was found to be significant at each of the other five combinations of cue duration and SOA (all $p$ s $<.005$ or less).

\section{EXPERIMENT 2}

The findings obtained in Experiment 1 serve to resolve an important empirical discrepancy that has arisen between studies that have reported null effects of uninformative arrow cues (Jonides, 1981) and those that have reported significant effects (Hommel et al., 2001; Ristic et al., 2002; Tipples, 2002). However, although the present findings suggest that this discrepancy may be accounted for in terms of cue duration and SOA, it is important to note that the specific nature of the cue duration $\times$ validity interaction that was observed in Experiment 1 remains to be addressed. One critical issue concerns whether stimulus-based or strategic-based factors may have caused the smaller cue validity effect in the $25-\mathrm{msec}$ cue duration condition than in the 200-msec cue duration condition. For instance, with respect to stimulus-based factors, the 25 -msec cues may have been more difficult to identify and/or easier to ignore than the $200-\mathrm{msec}$ cues (given constant effort). Alternatively, with respect to strategic-based factors, the observers may have tried harder to ignore the $25-\mathrm{msec}$ cues than the $200-\mathrm{msec}$ cues, or they may have used different strategies to ignore the 25 -msec cues than they did to ignore the $200-\mathrm{msec}$ cues. In Experiment 1, stimulus-based and strategicbased factors could not be readily distinguished, because the 25- and the 200-msec cues were presented in separate blocks of trials. Accordingly, we attempted to differentiate between these two classes of explanation by randomly mixing the two cue duration conditions within a single block of trials in Experiment 2. If the cue duration $\times$ validity interaction observed in Experiment 1 was due to stimulus-based factors, this interaction should remain in Experiment 2, because the same stimulus factors prevailed in this experiment. However, if the cue duration $\times$ validity interaction was due to strategic-based factors, this interaction should be eliminated under the present conditions, because the observers could no longer selectively apply different strategies (or the same strategy with different intensities) to the two cue conditions. 


\begin{abstract}
Method
Participants. Forty different undergraduates from the University of Notre Dame participated in this experiment in partial fulfillment of a course requirement. All the participants in the study reported normal or corrected-to-normal vision. None was aware of the purpose of the study. The data from one of the participants was dropped from the analyses because the error rate exceeded $20 \%$.

Stimuli and Apparatus. The stimuli and apparatus were identical to those in Experiment 1.

Procedure. The procedure was identical to that in Experiment 1 with the sole exception being that the 25 - and the 200 -msec cues were now randomly mixed within the same block of trials.
\end{abstract}

\section{Results and Discussion}

Mean correct RTs are shown in Figure 3 as a function of cue duration, SOA, and validity. Error rates were determined in the same manner as in Experiment 1 and are listed in Table 2 as a function of cue duration, SOA, and validity. The error rates were relatively low in this experiment $(3.26 \%)$ and generally mirrored the RT findings, suggesting that, once again, the observers did not sacrifice accuracy for speed in this experiment.

Mean correct RT was analyzed using a three-way repeated measures ANOVA, as in Experiment 1. There was a main effect of SOA $\left[F(2,76)=10.337, M S_{\mathrm{e}}=4,168.13\right.$, $p<.0001]$, which indicated that RTs generally decreased as SOA increased. There was also a main effect of validity $\left[F(1,38)=41.94, M S_{\mathrm{e}}=8,588.52, p<.0001\right]$, which indicated that RTs were significantly shorter in the valid condition than in the invalid condition. Most important, although the cue validity effect was slightly smaller in the $25-\mathrm{msec}$ cue duration condition (48 msec) than in the $200-\mathrm{msec}$ cue duration condition (62 $\mathrm{msec})$, the cue duration $\times$ validity interaction did not approach significance in this experiment $\left[F(1,38)=1.16, M S_{\mathrm{e}}=\right.$ $5,379.22, p>.25]$. Hence, the present findings provide evidence that the cue duration $X$ validity interaction observed in Experiment 1 was not due entirely to stimulusbased factors and likely depended primarily on top-down strategic factors that somehow differed (either qualitatively

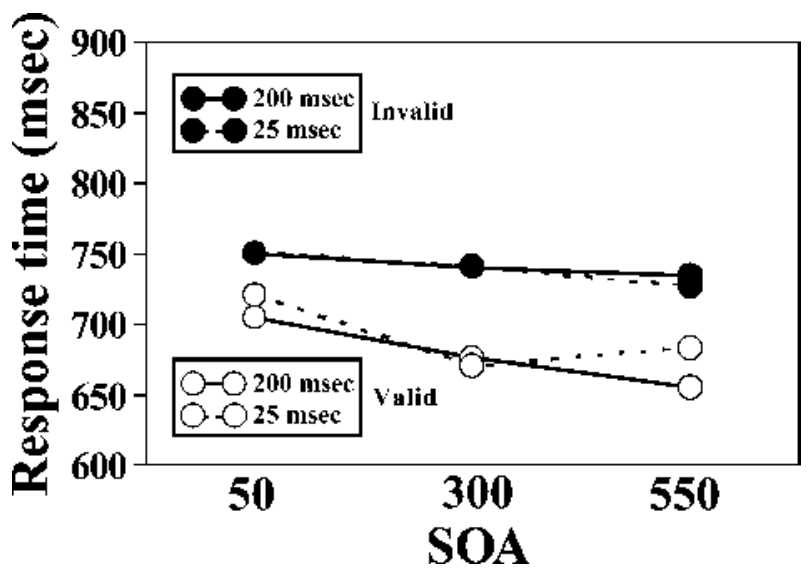

Figure 3. Mean correct response times shown as a function of cue duration, stimulus onset asynchrony (SOA, in milliseconds), and validity in Experiment 2.
Table 2

Error Rates (in Percentages) as a Function of

Cue Duration, Stimulus Onset Asynchrony

(SOA, in Milliseconds), and Validity in Experiment 2

\begin{tabular}{cccc}
\hline & \multicolumn{3}{c}{ SOA } \\
\cline { 2 - 4 } Validity & 50 & 300 & 550 \\
\hline & $25-\mathrm{msec}$ & Cue Duration \\
Valid & 2.56 & 3.30 & 2.93 \\
Invalid & 3.62 & 4.03 & 3.76 \\
& $200-\mathrm{msec}$ Cue Duration & \\
Valid & 1.60 & 2.93 & 1.60 \\
Invalid & 4.13 & 4.92 & 3.98 \\
\hline
\end{tabular}

or quantitatively) across trial blocks. In addition, in accord with Experiment 1, the cue validity effect increased in magnitude from 38 to $67 \mathrm{msec}$ as the cue-target SOA increased from 50 to $300 \mathrm{msec}$ and then remained relatively constant at $61 \mathrm{msec}$ as SOA increased further to $550 \mathrm{msec}$; however, unlike in Experiment 1, the SOA $\times$ validity interaction was found to be only marginally significant in the present experiment $[F(2,76)=2.14$, $\left.M S_{\mathrm{e}}=4,207.87, p<.13\right]$.

\section{EXPERIMENT 3}

The findings obtained in the first two experiments suggest that the relative magnitudes of the cue validity effects observed across the 25- and 200-msec conditions can vary as a function of blocking. When the two cues were presented in separate blocks, the cue validity effect was significantly smaller in the 25 -msec condition than in the 200-msec condition; however, when the two cues were randomly mixed within the same block, the magnitude of the cue validity effect did not differ significantly between the two cue conditions. Such a pattern suggests that the observed cue validity effects can be modulated by top-down factors (cf. Yantis \& Jonides, 1990). Experiment 3 was designed to provide further evidence for top-down factors, using a different experimental approach. Although the random mixing procedure used in Experiment 2 may have served to eliminate potential strategic differences (qualitative or quantitative) that occurred across the two cue duration conditions when cue duration was blocked, such a procedure would not necessarily have eliminated the use of top-down strategies per se. Moreover, such a procedure could not reveal the nature of the strategy that led to the cue duration $\times$ validity interaction observed in Experiment 1. In the present experiment, we explicitly instructed all the observers to engage in the same strategy not only to eliminate strategic differences between the two cue duration conditions, but also to better understand the nature of the strategy that might have been used in Experiment 1 to modulate the effects of the cues. We therefore maintained the original block design used in Experiment 1 in order to measure the effects of this manipulation independently of other factors (such as mixing) that might 
have also served to eliminate strategic differences between the two cue duration conditions.

By hypothesis, a symbolic cue can elicit a shift of attention only after its shape and meaning have been accessed from stored representations. As a result, it is possible that the putative top-down modulation observed in Experiment 1 occurred because cue processing differed in some way across the two cue duration conditions. Accordingly, the standard cuing procedure was modified in Experiment 3 to encourage more consistent processing of the cue across the two cue duration conditions. This was accomplished by requiring the observers in the present experiment to discriminate between two types of symbolic cues (arrows and bars) before responding to the target display. In particular, the observers in Experiment 3 were instructed to perform the target discrimination task (as they did in Experiments 1 and 2) only if an arrow cue had preceded the target display. In contrast, the observers were instructed to refrain from responding to the target display if a bar cue had preceded the target display. Note that although the observers in Experiment 3 were required only to discriminate the cues on the basis of their shape, we assumed that in processing the shape of the cues, the specific meaning of each of the cues would also be accessed (see Besner \& Stolz, 1999, for a discussion of this issue). Note also that although the observers in Experiment 3 were required to discriminate the shape of the cues, they were nevertheless told that the specific direction indicated by the cues was uninformative and that they should ignore this aspect of the cues. If the cue duration $\times$ validity interaction observed in Experiment 1 involved differential processing of the cues across the two cue conditions, the basis for this interaction should be eliminated in the present experiment, and the cuing effect observed in the 25 -msec cue duration condition should now be similar to the cuing effect observed in the 200msec cue duration condition. However, if this interaction was rooted in some other processing difference, it should once again be observed in the present experiment.

\section{Method}

Participants. Forty different undergraduates from the University of Notre Dame participated in this experiment in partial fulfillment of a course requirement. All the participants in the study reported normal or corrected-to-normal vision. None was aware of the purpose of the study.

Stimuli and Apparatus. The stimuli and apparatus were identical to those in Experiment 1, with the sole exception being the addition of a bar cue. The bar cue was constructed by removing the head of the arrow. The resultant bar measured the same length and width as the original arrow but did not point in any specific direction.

Procedure. The procedure was identical to that in Experiment 1, with the sole exception being that the arrow cue now appeared on only $75 \%$ of the trials within a block; the bar cue appeared on the remaining $25 \%$ of the trials. As in Experiment 1, the validity of the arrow cue was only $12.5 \%$. The observers were instructed to respond (in the same manner as in Experiment 1) only if an arrow preceded the target display. In addition, although the appearance of the arrow was relevant to the response, the observers were also told that the specific direction indicated by the arrow was irrelevant, and they were explicitly instructed to ignore the cue's meaning. The total number of arrow cue trials shown in Experiment 3 was the same as that in Experiment 1; however, the overall number of practice (40) and experimental (480) trials increased by $25 \%$ in Experiment 3, to accommodate the inclusion of the bar trials. Cue duration was once again held constant within each of the two trial blocks, and block order was counterbalanced across observers.

\section{Results and Discussion}

Mean correct RTs obtained when the arrow cue preceded the target display are shown in Figure 4 as a function of cue duration, SOA, and validity. Error rates were determined in the same manner as in Experiments 1 and 2 and are listed in Table 3 as a function of cue duration, SOA, and validity. Once again, error rates were relatively low in this experiment $(4.13 \%)$ and did not differ significantly across conditions (all $p \mathrm{~s}>.05$ or more). Thus, the RT findings reported below do not appear to be qualified by a speed-accuracy tradeoff.

Before proceeding to these RT results, however, we will first consider the false alarm rates that occurred in response to the bar cue (see Table 4). False alarms occurred when a target discrimination response was emitted following a bar cue, and these responses were analyzed using a two-way repeated measures ANOVA with cue duration and SOA as the two within-subjects factors (validity could not be defined for the bar). As can be seen in Table 4, false alarms generally increased as the SOA between the appearance of the cue and the appearance of the target decreased $\left[F(2,78)=23.33, M S_{\mathrm{e}}=63.53, p<\right.$ .0001 , for the main effect of SOA]. Follow-up ANOVAs revealed that this main effect of SOA was due primarily to the high false alarm rate obtained in the 50-msec SOA condition, relative to the $300-$ and $550-\mathrm{msec}$ SOA conditions $(p<.0001$ for 50 vs. $300 \mathrm{msec} ; p<.0001$ for 50 vs. $550 \mathrm{msec} ; p>.15$ for 300 vs. $550 \mathrm{msec}$ ). Of course, this finding is not entirely surprising, given that the target display likely appeared in the 50-msec SOA condition before the observer had completely processed the identity of the cue and/or completely inhibited his or her

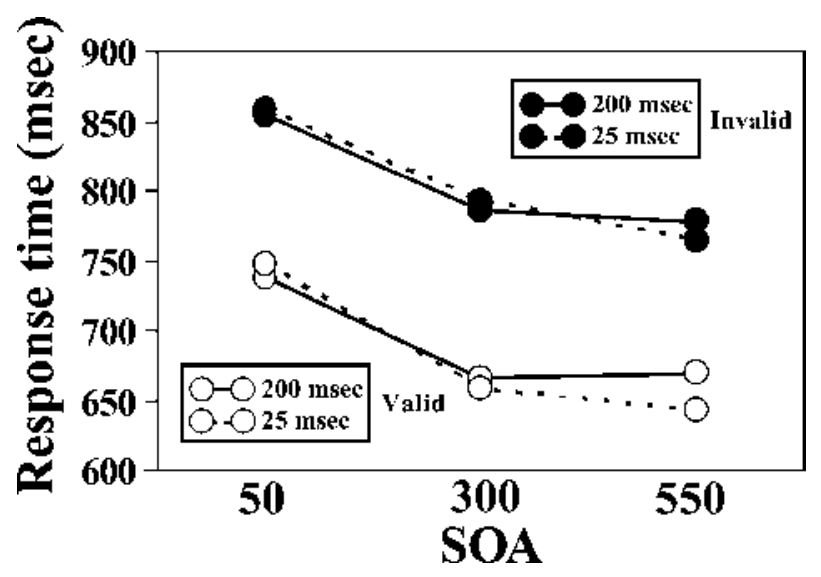

Figure 4. Mean correct response times obtained on go trials in Experiment 3, shown as a function of cue duration, stimulus onset asynchrony (SOA, in milliseconds), and validity. 
Table 3

Error Rates (in Percentages) as a Function of

Cue Duration, Stimulus Onset Asynchrony (SOA, in Milliseconds), and Validity in Experiment 3

\begin{tabular}{lccc}
\hline & \multicolumn{3}{c}{ SOA } \\
\cline { 2 - 4 } Validity & 50 & 300 & 550 \\
\hline \multirow{4}{*}{ Valid } & $25-\mathrm{msec}$ Cue Duration \\
Invalid & 2.93 & 3.30 & 2.56 \\
& 3.76 & 4.03 & 3.62 \\
Valid & $200-\mathrm{msec}$ Cue Duration & \\
Invalid & 1.33 & 2.93 & 1.60 \\
\hline
\end{tabular}

Table 4

False Alarm Rates on No-Go Trials as a Function of Cue Duration and Stimulus Onset Asynchrony (SOA, in Milliseconds) in Experiment 3

\begin{tabular}{cccc}
\hline & \multicolumn{3}{c}{ SOA } \\
\cline { 2 - 4 } Cue Duration (msec) & 50 & 300 & 550 \\
\hline 25 & 13.25 & 4.12 & 2.84 \\
200 & 9.60 & 5.28 & 3.97 \\
\hline
\end{tabular}

response. Even so, false alarms occurred on only $11.42 \%$ of the bar cue trials in the 50-msec SOA condition in this experiment. Moreover, although the pattern of false alarms was very similar across the two cue duration conditions, the rise in false alarms that occurred in the 50-msec SOA was steeper in the $25-\mathrm{msec}$ cue duration condition than in the 200-msec cue duration condition, resulting in a significant cue duration $\times$ SOA interaction $[F(2,78)=4.99$, $\left.M S_{\mathrm{e}}=30.73, p<.01\right]$. Altogether, the pattern of false alarm rates suggests that caution should be used when RT findings in the 50-msec SOA condition are interpreted. Nevertheless, we included this condition in the following RT analyses, since the findings obtained in this condition did not change, in any appreciable way, our interpretation of the data.

Turning now to the critical RT results, Figure 4 shows that there was a relatively large and consistent cue validity effect in both the 25- and the 200-msec cue duration conditions. Accordingly, there was a significant main effect of validity $\left[F(1,39)=110.09, M S_{\mathrm{e}}=15,367.13\right.$, $p<.0001]$, indicating that the RTs in the valid condition were significantly shorter than the RTs in the invalid condition. In addition, there was a significant main effect of SOA $\left[F(2,78)=55.26, M S_{\mathrm{e}}=6,239.07, p<\right.$ $.0001]$, which indicated that RTs generally decreased as SOA increased. Of particular interest was whether the methodology used in Experiment 3 would alter the interaction between cue duration and validity that was observed in Experiment 1. Contrary to the results of Experiment 1, the results of Experiment 3 showed that the magnitude of the cue validity effect was now virtually identical in both the 25- and the 200-msec cue duration conditions (122 and $115 \mathrm{msec}$, respectively), and as would be expected, the cue duration $\times$ validity interaction did not approach significance in this experiment $[F(1,39)<1]$.
In order to strengthen our conclusion that the cue discrimination task used in Experiment 3 caused a different pattern of cue validity effects from that observed in Experiment 1 , we repeated the analysis, including experiment as a between-groups factor. As was expected, there was a significant experiment $\times$ cue duration $\times$ validity interaction $\left[F(1,78)=4.18, M S_{\mathrm{e}}=5,102.21, p<.05\right]$, which indicated that the interaction between cue duration and validity did in fact differ significantly across experiments. Moreover, follow-up analyses revealed that the 122 -msec cue validity effect that was observed in the 25 -msec cue duration condition in Experiment 3 was significantly larger than the $42-\mathrm{msec}$ cue validity effect that was observed in Experiment $1\left[F(1,78)=23.21, M S_{\mathrm{e}}=\right.$ $8,397.18, p<.0001]$. Likewise, the 115 -msec cue validity effect that was observed in the $200-\mathrm{msec}$ cue duration condition in Experiment 3 was also found to be significantly larger than the $72-\mathrm{msec}$ cue validity effect observed in Experiment $1\left[F(1,78)=5.04, M S_{\mathrm{e}}=\right.$ $1,0949.26, p<.05]$. Altogether, the present findings are consistent with strategic-based factors and inconsistent with stimulus-based accounts, which would have continued to predict an interaction between cue duration and validity in the present context, due to the fact that the same stimulus factors prevailed in this experiment as in Experiment 1. In addition, the present findings also suggest that the cue discrimination task used in Experiment 3 encouraged more consistent processing of both the 25and the 200-msec cues, which in turn caused larger and more similar cue validity effects across the two cue duration conditions.

For the sake of completeness, it is also important to note that the magnitude of the cue validity effect was 115,128 , and $115 \mathrm{msec}$ in the 50-, 300-, and 550-msec SOA conditions, respectively; consequently, the interaction between SOA and validity did not approach significance in the present experiment $[F(2,78)<1]$. Thus, unlike in Experiments 1 and 2, the magnitude of the cue validity effect remained consistently large, regardless of the temporal interval between the appearance of the cue and the appearance of the target display. Recall that we interpreted the significant SOA $\times$ validity interaction observed in Experiment 1 as reflecting a constraint on the speed with which attention could be shifted from the central cue to the peripheral target display. In contrast, the present findings suggest that attention could be shifted equally well from the central cue to the peripheral target display, regardless of the temporal delay. Although such findings may be interpreted to suggest that attention was shifted more efficiently from the central cue to the peripheral target display in this experiment, such findings may have also been brought about by more efficient processing of the cue. In other words, it is likely that the observers processed the cue more efficiently in this experiment than in Experiment 1, given that they had to discriminate its category before they engaged in the visual search task. As a result, faster processing of the cue may have allowed sufficient time to optimally shift attention even at the shortest SOA in the present experiment. 


\section{GENERAL DISCUSSION}

The present study was conducted to investigate whether cue duration may have played a role in producing the disparate results observed across previous studies. The findings obtained in Experiment 1 showed that the duration of a symbolic cue did affect its ability to elicit involuntary shifts of attention. The influence of cue duration on involuntary orienting was reflected in the magnitude of the cue validity effect, which was $30 \mathrm{msec}$ smaller, on average, in the 25 -msec cue duration condition than in the 200-msec cue duration condition. Moreover, the findings obtained in Experiment 1 also showed that the magnitude of the cue validity effect increased as the SOA between the appearance of the cue and the appearance of the target increased from 50 to $300 \mathrm{msec}$. Altogether, the independent effects of cue duration and SOA on involuntary orienting that were observed in Experiment 1 are important because they can resolve the empirical discrepancy that has arisen between Jonides's (1981, Experiment 2) seminal study of involuntary orienting and more recent studies (Hommel et al., 2001; Ristic et al., 2002; Tipples, 2002). In particular, Experiment 1 provided a broad context in which a combination of both significant and null effects of indirect cuing could be observed. Within this broad context, some results corroborated the null effects reported by Jonides, but only when both cue duration and cue-target SOA were relatively brief ( 25 and $50 \mathrm{msec}$, respectively), whereas the results obtained in the remaining conditions were consistent with those in a variety of other recent studies that have collectively concluded that indirect cues can elicit involuntary shifts of attention (Hommel et al., 2001; Ristic et al., 2002; Tipples, 2002).

In addition to resolving this past empirical discrepancy, the present findings suggested that the difference in orienting that was observed across the two cue duration conditions in Experiment 1 was due primarily to top-down strategic factors, rather than to bottom-up stimulus factors. This conclusion followed, in part, from the results of Experiment 2, which showed that the magnitude of the cue validity effect in the 25 - and 200 -msec cue duration conditions was statistically equal when the two types of cues were randomly mixed within the same block of trials. In addition, the results of Experiment 3 corroborated this conclusion by showing that the interaction between cue duration and validity could also be eliminated by exerting greater control over which cue processing strategies observers might use when uninformative symbolic cues are shown. Hence, the present study provided strong evidence that the interaction observed between cue duration and validity in Experiment 1 can be attributed to topdown strategic factors, as opposed to bottom-up stimulus factors. These findings are important because they suggest that although uninformative symbolic cues may produce significant cue validity effects that are characteristic of involuntary shifts of attention, the relative magnitude of these effects may be modulated by top-down strategies.
In addition to providing evidence that the interaction between cue duration and validity in Experiment 1 was mediated by top-down strategies, the results of Experiment 3 provided suggestive evidence that this top-down modulation may have involved the application of different cue-processing strategies across the two cue duration conditions in Experiment 1. In particular, Experiment 3 showed that when the observers were required to discriminate the identity of uninformative arrow cues, both the 25- and the 200-msec cues produced equal (and relatively large) cue validity effects. In contrast, the observers in Experiment 1 had no cue-processing requirements and, in fact, were instructed only to ignore the cues. Under these conditions, the cues produced unequal (and relatively small) cue validity effects. Such a pattern points to differences in cue processing as a likely source of the observed cue duration $\times$ validity interaction in Experiment 1 . However, further research will be required to determine the precise nature of the mechanism underlying these different cue-processing strategies. Previous research has suggested that shape recognition processes may require attentional resources (Epstein \& Lovitts, 1985; Rock \& Gutman, 1981; Treisman \& Gelade, 1980), leading to the possibility that cue processing may be disrupted, at least to some degree, by withholding attentional resources from the centrally fixed cue. In addition, further research will be required to understand why cue processing varied as a function of cue duration in the present study. In other words, if cue processing can be disrupted by withholding attention from the cue, why would observers be more likely to withhold attention from 25-msec cues than from 200-msec cues? The present findings cannot provide a specific answer to this question; however, it is important to emphasize that the present findings do suggest that the differences in cue validity that were observed across cue durations reflect differences in strategy, not differences in cue duration. Thus, it is unlikely that the difference in cue validity that was observed across the 25- and 200-msec conditions in Experiment 1 occurred simply because the 25 -msec cues were easier to ignore than the 200-msec cues; otherwise, the same difference should have been observed in Experiment 2 as well.

Regardless of the nature of this top-down modulation, the present findings are important from a methodological standpoint, because they suggest that researchers may need to take extra precautions to ensure that adequate cue processing has occurred when uninformative symbolic cues are presented and observers are encouraged to ignore the cues. Of course, we acknowledge that significant cue validity effects can be obtained even when such precautions are not taken (see Experiments 1 and 2 of the present study; see also Eimer, 1997; Hommel et al., 2001; Ristic et al., 2002; Tipples, 2002); however, it is also important to realize that the failure to take such precautions may cause the observed cue validity effects to be underestimated (and perhaps differentially so across conditions), which in turn can generate misleading conclusions (especially when the 
use of such a strategy contributes to a null cue validity effect, as in Experiment 2 in Jonides, 1981).

In conclusion, the present findings have shown that the ability of an uninformative symbolic cue to elicit an involuntary shift of attention may depend on the extent to which the shape and meaning of the cue are processed. Moreover, the present findings have also suggested that observers may use top-down strategies to modulate the processing of symbolic cues when the cues are uninformative and the observers are instructed to ignore them, thus impacting the magnitude of the cue validity effect that is observed. As a result, we must remain mindful of the distinction between this form of involuntary orienting and the concept of stimulus-driven attentional capture that has arisen from direct cuing experiments over the past 2 decades (see Yantis, 1996, 1998, for reviews). Although both phenomena appear to involve involuntary forms of attentional orienting, the form elicited by indirect cues depends critically on access to stored representations of shape and meaning (and thus top-down sources of information), whereas the form elicited by direct cues does not. Thus, future research must be careful to distinguish between the mechanisms that underlie processing of the cue and the responses that follow from such processing. Of particular interest to this endeavor are recent memory-based theories of automaticity (e.g., Logan, 1988), which suggest that stimulus processing need not be performed preattentively in order to elicit an automatic - and presumably, involuntary - response (see also Hommel, 1998; Reynvoet, Caessens, \& Brysbaert, 2002). Rather, such responses often depend critically on the manner in which a stimulus is first attended and encoded (Logan \& Etherton, 1994). In addition, such responses often develop as a result of learning a consistent mapping between a particular stimulus and a particular response (Schneider \& Shiffrin, 1977). In the present study, the responses in question were orienting responses that are thought to occur more or less automatically following the recognition of an indirect cue. Future studies will be required to understand how the presumed automaticity of these responses develops over time as a result of learning, as well as the extent to which this form of involuntary orienting differs from other, more stimulusdriven forms of involuntary orienting.

\section{REFERENCES}

Bacon, W. F., \& Egeth, H. F. (1994). Overriding stimulus-driven attentional capture. Perception \& Psychophysics, 55, 485-496.

Besner, D., \& Stolz, J. A. (1999). What kind of attention modulates the Stroop effect? Psychonomic Bulletin \& Review, 6, 99-104.

Cheal, M., \& Lyon, D. (1991). Central and peripheral precuing of forced-choice discrimination. Quarterly Journal of Experimental Psychology, 43A, 859-880.

Driver, J., Davis, G., Ricciardelli, P., Kidd, P., Maxwell, E., \& BARON-CoHEN, S. (1999). Gaze perception triggers reflexive visuospatial orienting. Visual Cognition, 6, 509-540.

EIMER, M. (1997). Uninformative symbolic cues may bias visual-spatial attention: Behavioral and electrophysiological evidence. Biological Psychology, 46, 67-71.

EPstein, W., \& LovitTs, B. E. (1985). Automatic and attentional com- ponents in perception of shape-at-a-slant. Journal of Experimental Psychology: Human Perception \& Performance, 11, 355-366.

Folk, C., Remington, R., \& Johnston, J. (1992). Involuntary cover orienting is contingent on attentional control settings. Journal of Experimental Psychology: Human Perception \& Performance, 18, 1030-1044.

Friesen, C. K., \& Kingstone, A. (1998). The eyes have it! Reflexive orienting is triggered by nonpredictive gaze. Psychonomic Bulletin \& Review, 5, 490-495.

Friesen, C. K., \& Kingstone, A. (2003). Abrupt onsets and gaze direction cues trigger independent reflexive attentional effects. Cognition, 87, B1-B10.

Gibson, B. S., \& Kelsey, E. (1998). Stimulus-driven attentional capture is contingent on attentional set for displaywide visual features. Journal of Experimental Psychology: Human Perception \& Performance, 24, 699-706.

Hietanen, J. K. (1999). Does your gaze direction and head orientation shift my visual attention? NeuroReport, 10, 3443-3447.

Hommel, B. (1998). Event files: Evidence for automatic integration of stimulus-response episodes. Visual Cognition, 5, 183-216.

Hommel, B., Pratt, J., Colzato, L., \& Godijn, R. (2001). Symbolic control of visual attention. Psychological Science, 12, 360-365.

JONIDES, J. (1981). Voluntary versus automatic control over the mind's eye's movement. In J. [B.] Long \& A. [D.] Baddeley (Eds.), Attention and performance $I X$ (pp. 187-203). Hillsdale, NJ: Erlbaum.

Kingstone, A., Friesen, C. K., \& Gazzaniga, M. S. (2000). Reflexive joint attention depends on lateralized cortical connections. Psychological Science, 11, 159-165.

LogAN, G. D. (1988). Toward an instance theory of automatization. Psychological Review, 95, 492-527.

Logan G. D., \& Etherton, J. L. (1994). What is learned during automatization? The role of attention in constructing an instance. Journal of Experimental Psychology: Learning, Memory, \& Cognition, 20, $1022-1050$.

Müller, H. J., \& RabbitT, P. M. A. (1989). Reflexive and voluntary orienting of visual attention: Time course of activation and resistance to interruption. Journal of Experimental Psychology: Human Perception \& Performance, 15, 315-330.

PosNer, M. I. (1980). Orienting of attention. Quarterly Journal of Experimental Psychology, 32, 3-25.

Posner, M. I., \& Petersen, S. E. (1990). The attention system of the human brain. Annual Review of Neuroscience, 13, 25-42.

Posner, M. I., SNyder, C. R. R., \& Davidson, B. J. (1980). Attention and the detection of signals. Journal of Experimental Psychology: General, 109, 160-174.

Pratt, J., \& Hommel, B. (2003). Symbolic control of visual attention: The role of working memory and attentional control settings. Journal of Experimental Psychology: Human Perception \& Performance, 29, 835-845.

Reynyoet, B., Caessens, B., \& Brysbaert, M. (2002). Automatic stimulus-response associations may be semantically mediated. Psychonomic Bulletin \& Review, 9, 107-112.

Ristic, J., Friesen, C. K., \& Kingstone, A. (2002). Are the eyes special? It depends on how you look at it. Psychonomic Bulletin \& Review, 9, 507-513.

Rock, I., \& Gutman, D. (1981). The effect of inattention on form perception. Journal of Experimental Psychology: Human Perception \& Performance, 7, 275-285.

SCHNEIDER, W., \& SHIFFrIN, R. M. (1977). Controlled and automatic human information processing: I. Detection, search, and attention. Psychological Review, 84, 1-66.

Theeuwes, J. (1994). Stimulus-driven capture and attentional set: Selective search for color and visual abrupt onsets. Journal of Experimental Psychology: Human Perception \& Performance, 20, 799806.

Tipples, J. (2002). Eye gaze is not unique: Automatic orienting in response to uninformative arrows. Psychonomic Bulletin \& Review, 9, 314-318.

Treisman, A., \& Gelade, G. (1980). A feature integration theory of attention. Cognitive Psychology, 12, 97-136.

YANTIS, S. (1993). Stimulus-driven attentional capture and attentional 
control settings. Journal of Experimental Psychology: Human Perception \& Performance, 19, 676-681.

Yantis, S. (1996). Attentional capture in vision, In A. F. Kramer, M. G. H. Coles, \& G. D. Logan (Eds.), Converging operations in the study of visual selective attention (pp. 45-76). Washington, DC: American Psychological Association.

Yantis, S. (1998). Control of visual attention. In H. Pashler (Ed.), Attention (pp. 223-256). Hove, U.K.: Psychology Press/Erlbaum.
Yantis, S., \& Jonides, J. (1990). Abrupt visual onsets and selective attention: Voluntary versus automatic allocation. Journal of Experimental Psychology: Human Perception \& Performance, 16, 121-134.

(Manuscript received June 4, 2003;

revision accepted for publication September 13, 2004.) 